\title{
REVIEWERS OF THE „ENVIRONMENTAL PROTECTION AND NATURAL RESOURCES”
}

Vol. 27 No 1(67), 2(68), 3(69), 4(70), 2016

Prof. dr hab. Zygmunt Brogowski

Prof. dr hab. Stanisław Brożek

Dr Agnieszka Buczaj

Dr Jolanta Chmielewska-Badora

Prof. dr hab. Józef Chojnicki

Dr Monika Czop

Dr hab. Renata Gaj

Dr Magdalena Głogowska

Dr hab. Ewa Beata Górska

Dr hab. Andrzej Greinert, prof. nadzw.

Prof. dr hab. Barbara Gworek

Prof. dr hab. Mariusz Holtzer

Dr Krzysztof Iskra

Dr hab. Krzysztof Jóźwiakowski

Dr inż. Iwona Justyniak

Prof. dr hab. Cezary Kabała

Dr Radosław Kalinowski

Dr Beata Klojzy-Kaczmarczyk

Dr Agnieszka Kolada

Dr hab. Marek Kopacz, prof. nadzw.
Dr Bożena Kornatowska

Dr hab. Przemysław Kosobucki

Dr Mariusz Kuglarz

Dr Ewa Miszczak

Dr Damian Maciej Pisklak

Dr hab. Joanna Podlasińska

Dr Małgorzata Poniewozik

Dr hab. Grażyna Porębska, prof. nadzw.

Dr Ewa Puszczało

Dr Jadwiga Sienkiewicz

Prof. dr hab. Jerzy Siepak

Dr hab. Teresa Skrajna

Prof. dr hab. Ewa Spychaj-Fabisiak

Dr hab. Piotr Stachowski

DrŁukasz Szeleszczuk

Dr Anna Śliwińska-Wyrzychowska

Dr hab. Ligia Tuszyńska, prof. nadzw.

Dr hab. Bożena Wójtowicz, prof. nadzw.

Prof. dr hab. Jan Żarnowiec

Dr hab. Wioletta Żukiewicz-Sobczak, prof. nadzw.

The editors would like to publicly acknowledge the people who served as reviewers on the journal during 2016. Without their efforts, the quality of the journal could not be sustained. 